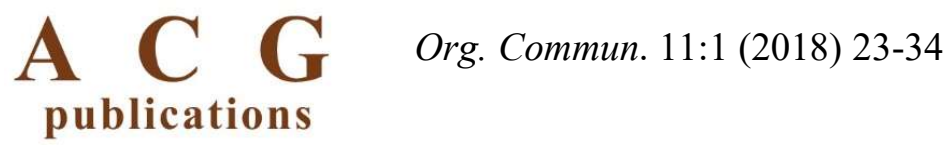

organic communications

\title{
Synthesis, theoretical calculation and $\alpha$-glucosidase inhibition of new chalcone oximes
}

\author{
Seda Fandaklı $\odot^{1}$, İnci Selin Doğan $\odot^{2}$, Hasan Erdinç Sellitepe $\odot^{2}$, \\ Ahmet Yaşar $\odot^{2}$ and Nurettin Yaylı $\odot^{* 2}$ \\ ${ }^{1}$ Department of Chemistry, Faculty of Science, Karadeniz Technical University, \\ 61080 Trabzon, Türkiye \\ ${ }^{2}$ Faculty of Pharmacy, Karadeniz Technical University, 61080 Trabzon, Türkiye
}

(Received February 01, 2018; Revised February 17, 2018; February 20, 2018)

\begin{abstract}
A series of eleven hydroxy and methoxy substituted new chalcone oximes (2a-2k) were synthesized by the condensation of chalcone (1a-1k) with hydroxylamine hydrochloride in pyridine. Structures of the synthesized compounds were characterized using NMR $\left(1 \mathrm{D} ;{ }^{1} \mathrm{H},{ }^{13} \mathrm{C} / \mathrm{APT}\right.$ and $2 \mathrm{D}{ }^{1} \mathrm{H}-{ }^{1} \mathrm{H}$ COSY, NOESY and ROESY), FT-IR, UV, LC-MS spectral data and elemental analysis. These synthetic compounds (2a-k) were screened for their $\alpha$-glucosidase inhibition. The most $\alpha$-glucosidase inhibitory activity were observe on compounds $2 \mathbf{a}$ and $\mathbf{2 b}$ with in the range of 1.61-3.36 $\mu \mathrm{M}$ ( $\mathrm{IC}_{50}$ values) which were more active then acarbose $\left(\mathrm{IC}_{50}, 13,34 \mu \mathrm{M}\right) . \quad \mathrm{IC}_{50}$ values of other synthesized compounds $\mathbf{2 c - 2} \mathbf{h}$ are within the range of 7,25-25,55 $\mu \mathrm{M}$ which were more or as active as acarbose, but, $\mathrm{IC}_{50}$ values for compounds $\mathbf{2} \mathbf{j}-\mathbf{2} \mathbf{k}$ were not observed. The geometric isomers of compounds $\mathbf{2 a - 2} \mathbf{k}$ were calculated theoretically. Experimental and theoretical calculations showed that cisoid $1 E, 2 E$ is the most stable geometrical isomer of all.
\end{abstract}

Keywords: Chalcone; chalcone oxime; $\alpha$-glucosidase inhibition. (c) 2018 ACG Publications. All rights reserved.

\section{Introduction}

$\alpha$-Glucosidase has a crucial role for the biosynthesis of glycoproteins and digestion of carbohydrates. ${ }^{1}$ Inhibition of enzyme plays an important role for the treatment of degenerative diseases. Some of $\alpha$-glucosidase inhibitors (Acarbose, Miglitol, and Voglibose) have been used as drugs by diabetic patients. ${ }^{2} \alpha$ - and $\beta$-glucosidases were known to catalyze the hydrolysis of the glycosidic bonds involving a terminal glucose at the cleavage site and they were most extensively studied. $^{3}$ Acarbose was the first member of $\alpha$-glucosidase inhibitors approved for the treatment of type II diabetes ${ }^{4}$. A number of $\alpha$-glucosidase inhibitors discovered recently and reviewed in an extensive fashion. ${ }^{5-7}$

Chalcones are medicinally important group of naturally occurring bioactive compounds. Chalcones and their analogues can be prepared by Claisen-Schmidt reaction and are well known intermediates for synthesizing various heterocyclic compounds. Chalcone possess various biological activities such as anti-tubercular, ${ }^{8-9}$ anti-inflammatory, ${ }^{10}$ antioxidant, ${ }^{11}$ antileishmanial, ${ }^{12}$ antimalarial, ${ }^{13-14}$ and antimicrobial. ${ }^{15-16}$ Since a broad spectrum of biological activities are associated with the natural or analogous chalcone compounds, and also its derivatives chalcone oximes and chalcone

\footnotetext{
* Corresponding author: E-mail: yayli@ktu.edu.tr; Phone: 0462337 8916; Fax: 04623256717

The article was published by ACG Publications

www.acgpubs.org/OC/ index.htm (C) January-March 2018 EISSN:1307-6175

DOI: http://doi.org/10.25135/acg.oc.38.18.02.067
} 
Schiff bases have been reported to exhibit various antimicrobial ${ }^{15-16}$ and tyrosinase inhibitory activities, ${ }^{17-18}$ respectively. As mentioned, many of the chalcone oxime compounds showed potent biological activities and low toxicities. ${ }^{19}$ Some of the chalcone oxime compounds have been used as clinical medical agents, thus, it was considered worthwhile to synthesize and evaluate a new series of chalcone oximes for their biological activity. The present study is devoted to the synthesis new hydroxy/methoxy substituted chalcone oximes $(\mathbf{2 a - 2} \mathbf{k})$ by the condensation of chalcones $(\mathbf{1} \mathbf{a}-\mathbf{1} \mathbf{k})$ with hydroxylamine hydrochloride in the pyridine and to test their $\alpha$-glucosidase inhibition activity and to find out the most stable geometric isomers of compounds $\mathbf{2 a - 2} \mathbf{k}$ by theoretical calculation. The synthesis of chalcone oxime starting form chalcone may have four possible geometric isomers. Theoretical calculations were performed with HYPERCHEM 7.5 program to determine the most stable geometric isomer of compounds $\mathbf{2} \mathbf{a}-\mathbf{2} \mathbf{k} .^{20-22}$ The compounds $\mathbf{2 g}$ and $\mathbf{2} \mathbf{j}$ had mentioned as an stereoisomer products with $1 Z, 2 E$ geometry in the literature with highly different melting points, ${ }^{23}$ but, in our case, geometry of double bonds for compounds $2 \mathbf{g}$ and $\mathbf{2} \mathbf{j}$ were found to be $1 E, 2 E$ geometry.

\section{Experimental}

\subsection{Materials and Apparatus}

All chemical reagents used in the synthesis were high grade of commercial products purchased from Sigma, Fluka or Aldrich and used without further purification. The solvents ( $n$-hexane, ethyl acetate, chloroform, diethyl ether, pyridine, methanol) used were either analytical grade or bulk solvents distilled before use. Thin-layer chromatography (TLC) and column chromatography were carried out on Merck precoated 60 Kieselgel $F_{254}$ analytical aluminum acidic plates and silica gel 60 $(0.040-0.063 \mathrm{~mm})$, respectively. Melting points were determined using Thermo-var apparatus fitted with a microscope and are uncorrected. ${ }^{1} \mathrm{H}$ and ${ }^{13} \mathrm{C}$ NMR spectra were recorded on a Bruker 400/100 $\mathrm{MHz}$ NMR with tetramethylsilane (TMS) as an internal standard, respectively. UV-Vis absorbance measurements and spectral analyses were carried out on a Unicam UV2-100 at $25{ }^{\circ} \mathrm{C}$. Infrared spectra were obtained with a Perkin-Elmer 1600 FT-IR $\left(4000-400 \mathrm{~cm}^{-1}\right)$ spectrometer. The mass spectral analyses were carried out on a Micromass Quattro LC-MS spectrophotometer. The elemental analyses were performed on a Costech ESC 4010 instrument.

\subsection{Methods}

The known chalcones ${ }^{19,24-31}$ (1a-1k) were synthesized according to the literature. ${ }^{32-33}$

\subsubsection{General procedure for the synthesis of chalcone oximes $\mathbf{2 a - 2} \mathbf{k}$ :}

Hydroxylamine hydrochloride $(0.01 \mathrm{~mol})$ in water $(5 \mathrm{~mL})$ was added to a solution of compound 1a-1k $(0.01 \mathrm{~mol})$ which were synthesized according to the literature in pyridine $(10 \mathrm{~mL}) \cdot{ }^{23,34-37}$ The reaction mixture was refluxed for $3 \mathrm{~h}$ with constant stirring and checked by TLC on silica gel plate. After the completion, the reaction mixture was cooled, acidified with diluted acetic acid, evaporated and purified with column chromatography using; hexane $50 \mathrm{~mL}, n$-hexane-ethyl acetate $(4: 1,50$ $\mathrm{mL} ; 4: 2,50 \mathrm{~mL} ; 1: 1,100 \mathrm{~mL}$ ) solvent system, respectively, to give compounds $\mathbf{2} \mathbf{a}-\mathbf{2} \mathbf{k}$.

(1E,2E)-1-(3-hydroxyphenyl)-3-phenylprop-2-en-1-one oxime (2a): Yield: 67\%, white solid, $R_{\mathrm{f}}$ $=0.67$ ( $n$-hexane-diethyl ether, 1:3); m.p. $\left({ }^{\circ} \mathrm{C}\right): 160-163$, UV-vis $\lambda \mathrm{nm}(\log \epsilon): 281.86(2.01), \mathrm{IR}(\mathrm{KBr}$, $\left.\mathrm{cm}^{-1}\right): 3262,3163(-\mathrm{OH},=\mathrm{NOH}), 1602(-\mathrm{C}=\mathrm{N}), 1594(-\mathrm{C}=\mathrm{C}$-olefinic $), 1574,1446(-\mathrm{C}=\mathrm{C}-$ aromatic $)$, ${ }^{1} \mathrm{H}-\mathrm{NMR}\left(400 \mathrm{MHz}, \mathrm{DMSO}-\mathrm{d}_{6}\right): 6.77$ (d, $\left.J: 16.0 \mathrm{~Hz}, 1 \mathrm{H}, \alpha-\mathrm{H}\right), 7.72(\mathrm{~d}, J: 16.0 \mathrm{~Hz}, 1 \mathrm{H}, \beta-\mathrm{H})$, ar-H: [7.49-7.46 (m, 2H), 7.37-7.25 (m, 5H), 6.79-6.75 (m, 2H)], ${ }^{13} \mathrm{C}-\mathrm{NMR}\left(100 \mathrm{MHz}, \mathrm{DMSO}-\mathrm{d}_{6}\right): 157.17$ ($\mathrm{C}=\mathrm{NOH}), 115.66(\alpha-\mathrm{C}), 138.55(\beta-\mathrm{C})$, ar-C [157.09 (C), $136.43(\mathrm{C}), 136.30(\mathrm{C}), 129.08(\mathrm{CH}), 128.75$ $(\mathrm{CH}), 128.50(2 \mathrm{CH}), 126.98(2 \mathrm{CH}), 120.22(\mathrm{CH}), 116.95(\mathrm{CH}), 115.81(\mathrm{CH})$, Anal. calcd for $\mathrm{C}_{15} \mathrm{H}_{13} \mathrm{NO}_{2}$ (m.w.: 239): C, 75.30; H, 5.48; N, 5.85. Found: C, 75.43; H, 5.56; N, 5.95. LC-MS: $(\mathrm{m} / \mathrm{z})$ $(\%)[\mathrm{M}-\mathrm{OH}+1]^{+}: 223(100)$. 
(1E,2E)-1-(4-hydroxyphenyl)-3-phenylprop-2-en-1-one oxime (2b): Yield: $69 \%$, white solid, $R_{\mathrm{f}}$ $=0.64$ ( $n$-hexane -diethyl ether, 1:3), m.p. $\left({ }^{\circ} \mathrm{C}\right): 156-159, \mathrm{UV}$-vis $\lambda \mathrm{nm}(\log \epsilon): 280.86(1.25), \mathrm{IR}(\mathrm{KBr}$, $\left.\mathrm{cm}^{-1}\right): 3268,3163(-\mathrm{OH},=\mathrm{NOH}), 1608(-\mathrm{C}=\mathrm{N}), 1594(-\mathrm{C}=\mathrm{C}$-olefinic $), 1575,1445(-\mathrm{C}=\mathrm{C}$-aromatic $)$, ${ }^{1} \mathrm{H}-\mathrm{NMR}\left(400 \mathrm{MHz}, \mathrm{DMSO}-\mathrm{d}_{6}\right): 6.79(\mathrm{~d}, J: 16.8 \mathrm{~Hz}, 1 \mathrm{H}, \alpha-\mathrm{H}), 7.62(\mathrm{~d}, J: 16.8 \mathrm{~Hz}, 1 \mathrm{H}, \beta-\mathrm{H}), 11.44$ $(\mathrm{s}, 1 \mathrm{H},=\mathrm{NOH}), 9.75(\mathrm{~s}, 1 \mathrm{H},-\mathrm{OH})$, ar-H: $\left[6.90-6.89(\mathrm{~m}, 2 \mathrm{H}),(7.42-7.35(\mathrm{~m}, 7 \mathrm{H})],{ }^{13} \mathrm{C}-\mathrm{NMR}(100\right.$ MHz, DMSO-d $\left.)_{6}\right): 157.98(-\mathrm{C}=\mathrm{NOH}), 117.56(\alpha-\mathrm{C}), 138.89(\beta-\mathrm{C})$, ar-C [157.58 (C), $136.31(\mathrm{C})$, $130.44(2 \mathrm{CH}), 128.81(\mathrm{CH}), 128.54(2 \mathrm{CH}), 127.18(2 \mathrm{CH}), 126.19(\mathrm{C}), 114.97(2 \mathrm{CH})]$. Anal. calcd for $\mathrm{C}_{15} \mathrm{H}_{13} \mathrm{NO}_{2}$ (m.w.: 239): C, 75.30; H, 5.48; N, 5.85, Found: 75.48; H, 5.59; N, 5.75. LC-MS: $(\mathrm{m} / \mathrm{z})(\%)$ $\left[\mathrm{M}+\mathrm{Na}+\mathrm{H}_{2} \mathrm{O}\right]^{+}: 280(100)$.

(1E,2E)-1,3-bis(2-methoxyphenyl)prop-2-en-1-one oxime (2c): Yield: 76\%, white solid, $R_{\mathrm{f}}=0.51$ ( $n$-hexane -diethyl ether, 1:3), m.p. $\left({ }^{\circ} \mathrm{C}\right): 126-128$, UV-vis $\lambda$ nm (loge): 283.45 (1,29), 343.77 (0.80), IR $\left(\mathrm{KBr}, \mathrm{cm}^{-1}\right): 3165(=\mathrm{NOH}), 1606(-\mathrm{C}=\mathrm{N}), 1593(-\mathrm{C}=\mathrm{C}$-olefinic $), 1487,1433(-\mathrm{C}=\mathrm{C}$-aromatic $),{ }^{1} \mathrm{H}-$ NMR (400 MHz, DMSO-d $)$ ): $11.31(\mathrm{~s}, 1 \mathrm{H},=\mathrm{NOH}), 6.74(\mathrm{~d}, J: 16.4 \mathrm{~Hz}, 1 \mathrm{H}, \alpha-\mathrm{H}), 7.76(\mathrm{~d}, J: 16.4 \mathrm{~Hz}$, $1 \mathrm{H}, \beta-\mathrm{H})$, ar-H: [7.61-7.59 (m, 2H), 7.40-7.19 (m, 4H), 7.05-6.92 (m, 2H)], 3,69, 3,68 (s, 6H, $\left.\mathrm{OCH}_{3}\right),{ }^{13} \mathrm{C}-\mathrm{NMR}\left(100 \mathrm{MHz}, \mathrm{DMSO}-\mathrm{d}_{6}\right): 163.96(-\mathrm{C}=\mathrm{NOH}), 120.79(\alpha-\mathrm{C}), 138.67(\beta-\mathrm{C})$, ar-C [161.21(C), $157.45(\mathrm{C}), 131.41(\mathrm{CH}), 130.20(\mathrm{CH}), 127.87(\mathrm{CH}), 127.92(\mathrm{CH}), 120.66(\mathrm{C}), 120.54$ (C), $126.09(\mathrm{CH}), 120.66(\mathrm{CH}), 117.35(\mathrm{CH}), 110.97(\mathrm{CH})$ ], 55.70, 55.48 [- $\left.-\mathrm{OCH}_{3}\right]$. Anal. calcd for $\mathrm{C}_{17} \mathrm{H}_{17} \mathrm{NO}_{3}$ (m.w.: 283): C, 72.07; H, 6.05; N, 4.94. Found: C, 72.08; H, 6.14; N, 4.91. LC-MS: $(\mathrm{m} / z)$ $(\%)[\mathrm{M}-\mathrm{OH}]^{+}: 266(100),[\mathrm{M}+\mathrm{H}]^{+}: 284(80)$.

(1E,2E)-3-(2,3-dimethoxyphenyl)-1-(2-methoxyphenyl)prop-2-en-1-one oxime (2d): Yield: $81 \%$, white solid, $R_{\mathrm{f}}=0.54$ ( $n$-hexane -diethyl ether, $\left.1: 3\right)$, m.p. $\left({ }^{\circ} \mathrm{C}\right): 121-123$, UV-vis $\lambda \mathrm{nm}(\log \epsilon)$ : 304.14 (1.41), IR $\left(\mathrm{KBr}, \mathrm{cm}^{-1}\right): 3184(=\mathrm{NOH}), 1599(-\mathrm{C}=\mathrm{N}), 1581(-\mathrm{C}=\mathrm{C}$-olefinic), 1593, 1433 (-C=Caromatic), ${ }^{1} \mathrm{H}-\mathrm{NMR}\left(400 \mathrm{MHz}, \mathrm{DMSO}-\mathrm{d}_{6}\right): 11.42(\mathrm{~s}, 1 \mathrm{H},=\mathrm{NOH}), 6.61(\mathrm{~d}, J: 16.8 \mathrm{~Hz}, 1 \mathrm{H}, \alpha-\mathrm{H}), 7.62$ $(\mathrm{d}, J: 16.8 \mathrm{~Hz}, 1 \mathrm{H}, \beta-\mathrm{H}), \quad$ ar- $\mathrm{H}:[7.43-7.47(\mathrm{~m}, 1 \mathrm{H}), 7.19-7.25(\mathrm{~m}, 2 \mathrm{H}), 6.99-6.14(\mathrm{~m}, 4 \mathrm{H})], 3.77$, 3.52, $3.50\left(\mathrm{~s}, 9 \mathrm{H},-\mathrm{OCH}_{3},{ }^{13}{ }^{3} \mathrm{C}-\mathrm{NMR}\left(100 \mathrm{MHz}, \mathrm{DMSO}-\mathrm{d}_{6}\right): 157.94(-\mathrm{C}=\mathrm{NOH}), 118.19(\alpha-\mathrm{C}), 131.45\right.$ $(\beta-\mathrm{C})$, ar-C [153.18 (C), $154.88(\mathrm{C}), 147.31(\mathrm{C}), 130.67(\mathrm{CH}), 130.40(\mathrm{CH}), 130.37(\mathrm{C}), 124.92(\mathrm{CH})$, $124.76(\mathrm{C}), 120.79(\mathrm{CH}), 118.19(\mathrm{CH}), 113.57(\mathrm{CH}), 111.83(\mathrm{CH})], 60.94,56.14,55.83\left[-\mathrm{OCH}_{3}\right]$. Anal. calcd for: $\mathrm{C}_{18} \mathrm{H}_{19} \mathrm{NO}_{4}$ (m.w.: 313): C, 68.99; H, 6.11; N, 4.47. Found: C, 68.82; H 6.21; N 4.43. LC-MS : $(\mathrm{m} / \mathrm{z})(\%)[\mathrm{M}+\mathrm{Na}]^{+}: 336(100)$.

(1E,2E)-1-(2-methoxyphenyl)-3-(2,3,4-trimethoxyphenyl)prop-2-en-1-one oxime (2e): Yield: $80 \%$, white solid, $R_{\mathrm{f}}=0.61$ ( $n$-hexane/diethyl ether, $\left.1: 3\right)$, m.p. $\left({ }^{\circ} \mathrm{C}\right): 154-156$, UV-vis $\lambda \mathrm{nm}(\log \mathrm{c})$ : 304.28 (1.53), IR $\left(\mathrm{KBr}, \mathrm{cm}^{-1}\right): 3178(=\mathrm{NOH}), 1598(-\mathrm{C}=\mathrm{N}), 1584(-\mathrm{C}=\mathrm{C}$-olefinic $), 1433(-\mathrm{C}=\mathrm{C}-$ aromatic), ${ }^{1} \mathrm{H}-\mathrm{NMR}\left(400 \mathrm{MHz}, \mathrm{DMSO}-\mathrm{d}_{6}\right): 11.02(\mathrm{~s}, 1 \mathrm{H},=\mathrm{NOH}), 6.62(\mathrm{~d}, J: 16.8 \mathrm{~Hz}, 1 \mathrm{H}, \alpha \mathrm{CH}), 7.67$ $(\mathrm{d}, J: 16.8 \mathrm{~Hz}, 1 \mathrm{H}, \beta \mathrm{CH})$, ar-H: [ 7.43-7.47 (m, 2H), 7.11-7.04 (m, 2H), 6.78-6.69 (m, 2H)], 3.91, 3.84, 3.74, $3.72\left(\mathrm{~s}, 12 \mathrm{H},-\mathrm{OCH}_{3},\right){ }^{13} \mathrm{C}-\mathrm{NMR}\left(100 \mathrm{MHz}, \mathrm{DMSO}-\mathrm{d}_{6}\right): 157.98(-\mathrm{C}=\mathrm{NOH}), 120.79(\alpha \mathrm{CH})$, $135.98(\beta \mathrm{CH})$, ar-C [156.56 (C), $155.57(\mathrm{C}), 154.48(\mathrm{C}), 133.32(\mathrm{CH}), 136.57(\mathrm{C}), 129.80(\mathrm{CH})$, $124.68(\mathrm{C}), 122.26(\mathrm{C}), 128.55(\mathrm{CH}), 127.25(\mathrm{CH}), 117.57(\mathrm{CH}), 112.08(\mathrm{CH})$ ], 61.31, 60.93, 56.21, 56.03, $55.83\left[-\mathrm{OCH}_{3}\right]$. Anal. calcd for $\mathrm{C}_{19} \mathrm{H}_{21} \mathrm{NO}_{5}$ (m.w.: 343): C, 66.46; H, 6.16; N, 4.08. Found: $\mathrm{C}$, 66.57; H, 6.21; N, 4.03. LC-MS: $(\mathrm{m} / \mathrm{z})(\%)[\mathrm{M}+1]^{+}: 344(100),[\mathrm{M}+\mathrm{Na}]^{+}: 366(75)$.

(1E,2E)-3-(2-methoxyphenyl)-1-(4-methoxyphenyl)prop-2-en-1-one oxime (2f): Yield: 72\%, white solid, $R_{\mathrm{f}}=0.57$ ( $n$-hexane-diethyl ether, 1:3), m.p. $\left({ }^{\circ} \mathrm{C}\right)$ : $134-135$, UV-vis $\lambda \mathrm{nm}(\log \epsilon): 279.27$ (1.50), 321.30 (1.04), IR $\left(\mathrm{KBr}, \mathrm{cm}^{-1}\right): 3162(=\mathrm{NOH}), 1608(-\mathrm{C}=\mathrm{N}), 1594(-\mathrm{C}=\mathrm{C}-$ olefinic $), 1577$, 1447(-C=C-aromatic), ${ }^{1} \mathrm{H}-\mathrm{NMR}\left(400 \mathrm{MHz}, \mathrm{DMSO}-\mathrm{d}_{6}\right): 11.51(\mathrm{~s}, 1 \mathrm{H},=\mathrm{NOH}), 6.90(\mathrm{~d}, J: 16.0 \mathrm{~Hz}, 1 \mathrm{H}$, $\alpha-\mathrm{H}), 7.69(\mathrm{~d}, J: 16.0 \mathrm{~Hz}, 1 \mathrm{H}, \beta-\mathrm{H})$, ar-H: [7.47-7.51 (m, 2H), 7.33-7.37 (m, 2H), 6.99-6.88 (m, 4H)], 3.88, $3.83\left(\mathrm{~s}, 6 \mathrm{H},-\mathrm{OCH}_{3},\right),{ }^{13} \mathrm{C}-\mathrm{NMR}\left(100 \mathrm{MHz}, \mathrm{DMSO}-\mathrm{d}_{6}\right): 160.38(-\mathrm{C}=\mathrm{NOH}), 117.77(\alpha-\mathrm{C}), 134.58$ $(\beta-\mathrm{C})$, ar-C [157.61(C), 157.87(C), $130.54(\mathrm{CH}), 130.54(\mathrm{CH}), 130.24(\mathrm{CH}), 128.74(\mathrm{CH}), 127.45(\mathrm{C})$, $127.64(\mathrm{CH}), 125.35(\mathrm{C}), 113.81(\mathrm{CH}), 113.72(\mathrm{CH}), 110.98(\mathrm{CH})], 55.58,55.42,\left[-\mathrm{OCH}_{3}\right] . \mathrm{LC} / \mathrm{MS}$ : $(\mathrm{m} / \mathrm{z})(\%)[\mathrm{M}+1]: 268$ (100). Anal. calcd for $\mathrm{C}_{17} \mathrm{H}_{17} \mathrm{NO}_{3}$ (m.w.: 283): C, 72.07; H, 6.05; N, 4.94. 
Found: C, 72.12; H, 5.98; N, 5.03. LC-MS: $(\mathrm{m} / \mathrm{z}) \%[\mathrm{M}+\mathrm{K}-\mathrm{H}]^{+}: 321(100),[\mathrm{M}+\mathrm{K}]^{+}:$322(13), $\left[\mathrm{M}+\mathrm{Na}+\mathrm{H}_{2} \mathrm{O}+\mathrm{H}\right]^{+}: 325(50)$.

(1E,2E)-3-(3-methoxyphenyl)-1-(4-methoxyphenyl)prop-2-en-1-one oxime (2g): Yield: 74\%, white solid, $R_{\mathrm{f}}=0.59$ (n-hexane-diethyl ether, 1:3), m.p. $\left({ }^{\circ} \mathrm{C}\right): 113-114$, UV-vis $\lambda \mathrm{nm}(\log \epsilon)$ : 284.41(0.96), IR $\left(\mathrm{KBr}, \mathrm{cm}^{-1}\right): 3160(=\mathrm{NOH}), 1603(-\mathrm{C}=\mathrm{N}), 1578(-\mathrm{C}=\mathrm{C}-$ olefinic $), 1513,1440$ (-C=Caromatic) ${ }^{1}$ H-NMR $\left(400 \mathrm{MHz}, \mathrm{DMSO}-\mathrm{d}_{6}\right): 6.55(\mathrm{~d}, J: 15.6 \mathrm{~Hz}, 1 \mathrm{H}, \alpha-\mathrm{H}), 7.68(\mathrm{~d}, J: 15.6 \mathrm{~Hz}, 1 \mathrm{H}, \beta-$ $\mathrm{H})$, ar-H: $[7.47-7.51(\mathrm{~m}, 2 \mathrm{H}), 7.36-7.27(\mathrm{~m}, 1 \mathrm{H}), 6.99-6.86(\mathrm{~m}, 5 \mathrm{H})], 3.89,3.84\left(\mathrm{~s}, 6 \mathrm{H},-\mathrm{OCH}_{3}\right){ }^{13} \mathrm{C}-$ NMR $\left(100 \mathrm{MHz}, \mathrm{DMSO}-\mathrm{d}_{6}\right): 160.47(-\mathrm{C}=\mathrm{NOH}), 117.66(\alpha-\mathrm{C}), 139.49(\beta-\mathrm{C})$, ar-C [159.89 (C), $157.33(\mathrm{C}), 130.50(\mathrm{CH}), 137.52(\mathrm{C}), 130.54(\mathrm{CH}), 130.24(\mathrm{CH}), 129.72(\mathrm{CH}), 120.27(\mathrm{CH}), 126.52$ (C), $113.83(\mathrm{CH}), 113.91(\mathrm{CH}), 112.30(\mathrm{CH})], 55.58,55.42,\left[-\mathrm{OCH}_{3}\right] . \quad \mathrm{LC} / \mathrm{MS}:(\mathrm{m} / \mathrm{z})(\%)[\mathrm{M}+1]$ : 268 (100). Anal. calcd for $\mathrm{C}_{17} \mathrm{H}_{17} \mathrm{NO}_{3}$ (m.w.: 283): C, 72.07; H, 6.05; N, 4.94. Found: C, 71.94; H, 5.96; N, 5.05. LC-MS: $(\mathrm{m} / \mathrm{z})(\%)[\mathrm{M}-\mathrm{OH}]^{+}: 266(85),[\mathrm{M}+1]: 284(40)$.

(1E,2E)-3-(2,3-dimethoxyphenyl)-1-(4-methoxyphenyl)prop-2-en-1-one oxime (2h): Yield: $73 \%$, white solid, $R_{\mathrm{f}}=0.72$ ( $n$-hexane-diethyl ether, 1:3), m.p. $\left({ }^{\circ} \mathrm{C}\right): 116-118$, UV-vis $\lambda \mathrm{nm}(\log \epsilon)$ : 305.57 (1.21), 326.14 (1.19), IR $\left(\mathrm{KBr}, \mathrm{cm}^{-1}\right): 3152(=\mathrm{NOH}), 1606(\mathrm{C}=\mathrm{N}), 1576(-\mathrm{C}=\mathrm{C}-$ olefinic $), 1514$, $1442\left(-\mathrm{C}=\mathrm{C}\right.$-aromatic), ${ }^{1} \mathrm{H}-\mathrm{NMR}\left(400 \mathrm{MHz}, \mathrm{DMSO}_{-} \mathrm{d}_{6}\right): 11.54(\mathrm{~s}, 1 \mathrm{H},=\mathrm{NOH}), 6.98(\mathrm{~d}, J: 16.8 \mathrm{~Hz}$, $1 \mathrm{H}, \alpha-\mathrm{H}), 7.67(\mathrm{~d}, J: 16.8 \mathrm{~Hz}, 1 \mathrm{H}, \beta-\mathrm{H}), \quad$ ar-H: $[7.35-7.29(\mathrm{~m}, 2 \mathrm{H}), 7,12-7,08(\mathrm{~m}, 3 \mathrm{H}) 7.02$ (d, $J: 8.0$ $\mathrm{Hz}, 2 \mathrm{H})], 3.41,3.56,3.62\left(\mathrm{~s}, 9 \mathrm{H}, \mathrm{OCH}_{3}\right),{ }^{13} \mathrm{C}-\mathrm{NMR}\left(100 \mathrm{MHz}, \mathrm{DMSO}-\mathrm{d}_{6}\right): 160.16(-\mathrm{C}=\mathrm{NOH}), 118.31$ $(\alpha-\mathrm{C}), 131.40(\beta-\mathrm{C})$, ar-C [157.00 (C), $155.64(\mathrm{C}), 147.44(\mathrm{C}), 130.25(2 \mathrm{CH}), 128.62(\mathrm{C}), 128.42(\mathrm{C})$, 124.66 $(\mathrm{CH}), 119.25(\mathrm{CH}), 124.66(\mathrm{CH}), 114.20(2 \mathrm{CH})], 61.08,56.13,55.62,\left[-\mathrm{OCH}_{3}\right]$. Anal. calcd for $\mathrm{C}_{18} \mathrm{H}_{19} \mathrm{NO}_{4}$ (m.w.: 313): C, 68.99; H, 6.11; N, 4.47. Found: C, 68.90; H, 6.27; N, 4.58. LC-MS: $(\mathrm{m} / \mathrm{z})(\%)[\mathrm{M}+1]^{+}: 314(100),[\mathrm{M}+\mathrm{Na}]^{+}: 336(55)$.

(1E,2E)-3-(2,4-dimethoxyphenyl)-1-(4-methoxyphenyl)prop-2-en-1-one oxime (2i): Yield: $74 \%$, white solid, $R_{\mathrm{f}}=0.66$ ( $n$-hexane-diethyl ether, 1:3), m.p. $\left({ }^{\circ} \mathrm{C}\right): 148-149$, UV-vis $\lambda \mathrm{nm}(\log \epsilon)$ : 245.30 (0.98), $312.12(1.21)$, IR $\left(\mathrm{KBr}, \mathrm{cm}^{-1}\right): 3151(=\mathrm{NOH}), 1606(-\mathrm{C}=\mathrm{C}-$ olefinic $), 1579(-\mathrm{C}=\mathrm{C}-$ aromatic), ${ }^{1} \mathrm{H}-\mathrm{NMR}\left(400 \mathrm{MHz}, \mathrm{DMSO}-\mathrm{d}_{6}\right): 6.96(\mathrm{~d}, J: 16.0 \mathrm{~Hz}, 1 \mathrm{H}, \alpha-\mathrm{H}), 7.59(\mathrm{~d}, J: 16.0 \mathrm{~Hz}, 1 \mathrm{H}, \beta-$ H), ar-H: [ 7.42-7.45 (m, 2H), 7.49-7.51 (m, 3H), 6.93-6.97 (m, 2H)], 3.80, 3.84, $3.87(\mathrm{~s}, 9 \mathrm{H},-$ $\left.\mathrm{OCH}_{3}\right),{ }^{13} \mathrm{C}-\mathrm{NMR}\left(100 \mathrm{MHz}, \mathrm{DMSO}-\mathrm{d}_{6}\right): 161.75(-\mathrm{C}=\mathrm{NOH}), 115.53(\alpha-\mathrm{C}), 134.40(\beta-\mathrm{C}), \quad$ ar-C [160.28 (C), $158.92(\mathrm{C}), 158,16(\mathrm{C}), 130.72(\mathrm{CH}), 130.57(\mathrm{CH}), 128.74(\mathrm{CH}), 127.66(\mathrm{C}), 118.48(\mathrm{C})$, $113.74(\mathrm{CH}), 113.69(\mathrm{CH}), 98.47(\mathrm{CH}), 106.12(\mathrm{CH})], 55.48,55.42,55.31\left[-\mathrm{OCH}_{3}\right]$. Anal. calcd for $\mathrm{C}_{18} \mathrm{H}_{19} \mathrm{NO}_{4}$ (m.w.: 313): C, 68.99; H, 6.11; N, 4.47. Found: C, 68.92; H, 6.21; N, 4.43. LC-MS: $(\mathrm{m} / \mathrm{z})$ (\%) $[\mathrm{M}-\mathrm{OH}]^{+}: 296(100),[\mathrm{M}+\mathrm{H}]^{+}: 314(90)$.

(1E,2E)-1-(4-methoxyphenyl)-3-(2,4,5-trimethoxyphenyl)prop-2-en-1-one oxime (2j): Yield: $66 \%$, white solid, $R_{\mathrm{f}}=0.63$ ( $n$-hexane-diethyl ether, 1:3), m.p. $\left({ }^{\circ} \mathrm{C}\right): 160-162$, UV-vis $\lambda \mathrm{nm}(\log \epsilon)$ : 285.20 (1.52), 294.28 (1.42), 349.01 (1.68), IR (KBr,cm $\left.{ }^{1}\right): 3157(=\mathrm{NOH}), 1604(\mathrm{C}=\mathrm{N}), 1568(-\mathrm{C}=\mathrm{C}-$ olefinic), 1510, 1436 (-C=C-aromatic), ${ }^{1} \mathrm{H}-\mathrm{NMR}\left(400 \mathrm{MHz}, \mathrm{DMSO}-\mathrm{d}_{6}\right): 11.34(\mathrm{~s}, 1 \mathrm{H},=\mathrm{NOH}), 6.65$ $(\mathrm{d}, J: 16.0 \mathrm{~Hz}, 1 \mathrm{H}, \alpha-\mathrm{H}), 7.60(\mathrm{~d}, J: 16.0 \mathrm{~Hz}, 1 \mathrm{H}, \beta-\mathrm{H})$, ar- $\mathrm{H}:[7.58-7.62(\mathrm{~m}, 2 \mathrm{H}), 7.15-7.17(\mathrm{~m}, 1 \mathrm{H})$, 6.98-7.03 (m, 2H), 6.67-6.68 (m, 1H)], 3.68, 3.73, 3.81, $3.83\left(\mathrm{~s}, 12 \mathrm{H},-\mathrm{OCH}_{3}\right),{ }^{13} \mathrm{C}-\mathrm{NMR}(100 \mathrm{MHz}$, DMSO-d $\left._{6}\right): 160.03(-\mathrm{C}=\mathrm{NOH}), 116.19(\alpha-\mathrm{C}), 131.64(\beta-\mathrm{C})$, ar-C [156.13 $(\mathrm{C}), 157.47(\mathrm{C}), 152.66(\mathrm{C})$, $151.87(\mathrm{C}), 130.55(2 \mathrm{CH}), 130.52(\mathrm{C}), 116.16(\mathrm{C}), 114.15(2 \mathrm{CH}), 110.47(\mathrm{CH}), 98.43(\mathrm{CH}).], 55.54$, 56.19, 56.64, $56.76\left(-\mathrm{OCH}_{3}\right)$. Anal. calcd for $\mathrm{C}_{19} \mathrm{H}_{21} \mathrm{NO}_{5}$ (m.w.: 343): C, 66.46; H, 6.16; N, 4.08 . Found: C, 66.56 ; H, 6.19; N, 4.12. LC-MS: $(\mathrm{m} / \mathrm{z})(\%)\left[\mathrm{M}+\mathrm{H}_{2} \mathrm{O}-1\right]^{+}: 360(100)$.

(1E,2E)-1-(4-methoxyphenyl)-3-(2,3,4-trimethoxyphenyl)prop-2-en-1-one oxime (2k): Yield: $67 \%$, white solid, $R_{\mathrm{f}}=0.74$ ( $n$-hexane-diethyl ether, 1:3), m.p. $\left({ }^{\circ} \mathrm{C}\right): 139-140$, UV UV-vis $\lambda \mathrm{nm}(\log \epsilon)$ : 246.30 (0.62), 312.12 (0.82), IR (KBr, cm $\left.{ }^{-1}\right): 3149$ (=NOH), $1601(-\mathrm{C}=\mathrm{N}), 1598$ (-C=C-olefinic), 1493 (-C=C-aromatic), ${ }^{1} \mathrm{H}-\mathrm{NMR}\left(400 \mathrm{MHz}, \mathrm{DMSO}_{6}\right): 11.34(\mathrm{~s}, 1 \mathrm{H},=\mathrm{NOH}), 6.87(\mathrm{~d}, \mathrm{~J}: 16.0 \mathrm{~Hz}, 1 \mathrm{H}, \alpha-$ H), $7.49(\mathrm{~d}, J: 16.0 \mathrm{~Hz}, 1 \mathrm{H}, \beta-\mathrm{H})$, ar-H: [7.40-7.43 (m, 3H), $7.01(\mathrm{~d}, 2 \mathrm{H}), 6.81-6.84(\mathrm{~m}, 1 \mathrm{H})], 3.68$, 3.73, 3.81, $3.83\left(\mathrm{~s}, 12 \mathrm{H}, \mathrm{OCH}_{3}\right),{ }^{13} \mathrm{C}-\mathrm{NMR}\left(100 \mathrm{MHz}, \mathrm{DMSO}-\mathrm{d}_{6}\right): 160.09(-\mathrm{C}=\mathrm{NOH}), 117.19(\alpha-\mathrm{C})$, 131.53 ( $\beta-\mathrm{C})$, ar-C [154.57 (C), $155.86(\mathrm{C}), 152.22(\mathrm{C}), 142.34(\mathrm{C}), 130.49(2 \mathrm{CH}), 128.28(\mathrm{C}), 123.06$ 
(C), $121.89(\mathrm{CH}), 114.16(2 \mathrm{CH}), 109.09(\mathrm{CH})], 55.63,56.41,60.89,61.67\left(-\mathrm{OCH}_{3}\right)$. Anal. calcd for $\mathrm{C}_{19} \mathrm{H}_{21} \mathrm{NO}_{5}$ (m.w.: 343): C, 66.46; H, 6.16; N, 4.08. Found: C, 66.57 ; H, 6.21; N, 4.03. LC-MS: $(\mathrm{m} / z)$ $(\%)[\mathrm{M}+\mathrm{Na}]^{+}: 366(100),[\mathrm{M}+\mathrm{Na}+\mathrm{H}]^{+}: 367(60)$.<smiles>[R10]c1ccc([R2])c(C=O)c1</smiles>

\begin{tabular}{lccccccc} 
Comp. & $\mathbf{R}_{\mathbf{1}}$ & $\mathbf{R}_{\mathbf{2}}$ & $\mathbf{R}_{\mathbf{3}}$ & $\mathbf{R}_{\mathbf{4}}$ & $\mathbf{R}_{\mathbf{5}}$ & $\mathbf{R}_{\mathbf{6}}$ & $\mathbf{R}_{7}$ \\
\hline $\mathbf{1 a}, \mathbf{2 a}$ & $-\mathrm{H}$ & $-\mathrm{OH}$ & $-\mathrm{H}$ & $-\mathrm{H}$ & $-\mathrm{H}$ & $-\mathrm{H}$ & $-\mathrm{H}$ \\
$\mathbf{1 b}, \mathbf{2 b}$ & $-\mathrm{H}$ & $-\mathrm{H}$ & $-\mathrm{OH}$ & $-\mathrm{H}$ & $-\mathrm{H}$ & $-\mathrm{H}$ & $-\mathrm{H}$ \\
$\mathbf{1 c}, \mathbf{2 c}$ & $-\mathrm{OCH}_{3}$ & $-\mathrm{H}$ & $-\mathrm{H}$ & $-\mathrm{OCH}_{3}$ & $-\mathrm{H}$ & $-\mathrm{H}$ & $-\mathrm{H}$ \\
$\mathbf{1 d}, \mathbf{2 d}$ & $-\mathrm{OCH}_{3}$ & $-\mathrm{H}$ & $-\mathrm{H}$ & $-\mathrm{OCH}_{3}$ & $-\mathrm{OCH}_{3}$ & $-\mathrm{H}$ & $-\mathrm{H}$ \\
$\mathbf{1 e}, \mathbf{2 e}$ & $-\mathrm{OCH}_{3}$ & $-\mathrm{H}$ & $-\mathrm{H}$ & $-\mathrm{OCH}_{3}$ & $-\mathrm{OCH}_{3}$ & $-\mathrm{OCH}_{3}$ & $-\mathrm{H}$ \\
$\mathbf{1 f}, \mathbf{2 f}$ & $-\mathrm{H}$ & $-\mathrm{H}$ & $-\mathrm{OCH}_{3}$ & $-\mathrm{OCH}_{3}$ & $-\mathrm{H}$ & $-\mathrm{H}$ & $-\mathrm{H}$ \\
$\mathbf{1 g}, \mathbf{2 g}$ & $-\mathrm{H}$ & $-\mathrm{H}$ & $-\mathrm{OCH}_{3}$ & $-\mathrm{H}$ & $-\mathrm{OCH}_{3}$ & $-\mathrm{H}$ & $-\mathrm{H}$ \\
$\mathbf{1 h}, \mathbf{2 h}$ & $-\mathrm{H}$ & $-\mathrm{H}$ & $-\mathrm{OCH}_{3}$ & $-\mathrm{OCH}_{3}$ & $-\mathrm{OCH}_{3}$ & $-\mathrm{H}$ & $-\mathrm{H}$ \\
$\mathbf{1 i}, \mathbf{2 i}$ & $-\mathrm{H}$ & $-\mathrm{H}$ & $-\mathrm{OCH}_{3}$ & $-\mathrm{OCH}_{3}$ & $-\mathrm{H}$ & $-\mathrm{OCH}_{3}$ & $-\mathrm{H}$ \\
$\mathbf{1 j}, \mathbf{2 j}$ & $-\mathrm{H}$ & $-\mathrm{H}$ & $-\mathrm{OCH}_{3}$ & $-\mathrm{OCH}_{3}$ & $-\mathrm{H}$ & $-\mathrm{OCH}_{3}$ & $-\mathrm{OCH}_{3}$ \\
$\mathbf{1 k}, \mathbf{2 k}$ & $-\mathrm{H}$ & $-\mathrm{H}$ & $-\mathrm{OCH}_{3}$ & $-\mathrm{OCH}_{3}$ & $-\mathrm{OCH}_{3}$ & $-\mathrm{OCH}_{3}$ & $-\mathrm{H}$ \\
\hline
\end{tabular}

Scheme 1. Synthesis of chalcone oximes (2a-2k) from chalcones (1a-1k).

\section{3. $\alpha$-Glucosidase Inhibition Assay}

$\alpha$-Glucosidase inhibition assay was performed spectrophotometrically. $\alpha$-Glucosidase from Saccharomyces cerevisiae (Sigma-Aldrich) was dissolved in phosphate buffer (pH 6.8, $50 \mathrm{mM}$ ). Test compounds were dissolved in DMSO. In 96-well microtiter plates, $20 \mu \mathrm{L}$ of test sample, $20 \mu \mathrm{L}$ of enzyme $(20 \mathrm{mU} / \mathrm{mL})$ and $135 \mu \mathrm{L}$ of buffer were added and incubated for 15 minutes at $37^{\circ} \mathrm{C}$. After incubation, $25 \mu \mathrm{L}$ of $p$-nitrophenyl- $\alpha$-D-glucopyranoside $(2 \mathrm{mM}$, Sigma Aldrich) was added and change in absorbance was monitored for 30 minutes at $400 \mathrm{~nm}$. Test compound was replaced by DMSO (7.5\% final) as control. Acarbose (Sigma-Aldrich) was used as a standard inhibitor. ${ }^{35-39}$

\section{Result and Discussion}

\subsection{Synthesis}

In this work, due to the biological evaluation, known hydroxy and methoxy substituted chalcones ${ }^{19,24-31}$ (1a-1k) have been prepared by the Claisen-Schmidt condensation reaction by using substituted acetophenone and benzaldehyde ${ }^{32-33}$ (Scheme 1), then a new series of chalcone oximes (2a2k) were synthesized from corresponding chalcones (1a-1k) and hydroxyl amine hydrochloride at 
reflux temperature using pyridine as solvent in the range of $66-81 \%$ yield, respectively (scheme 1 ). The crude products were purified with column chromatography.

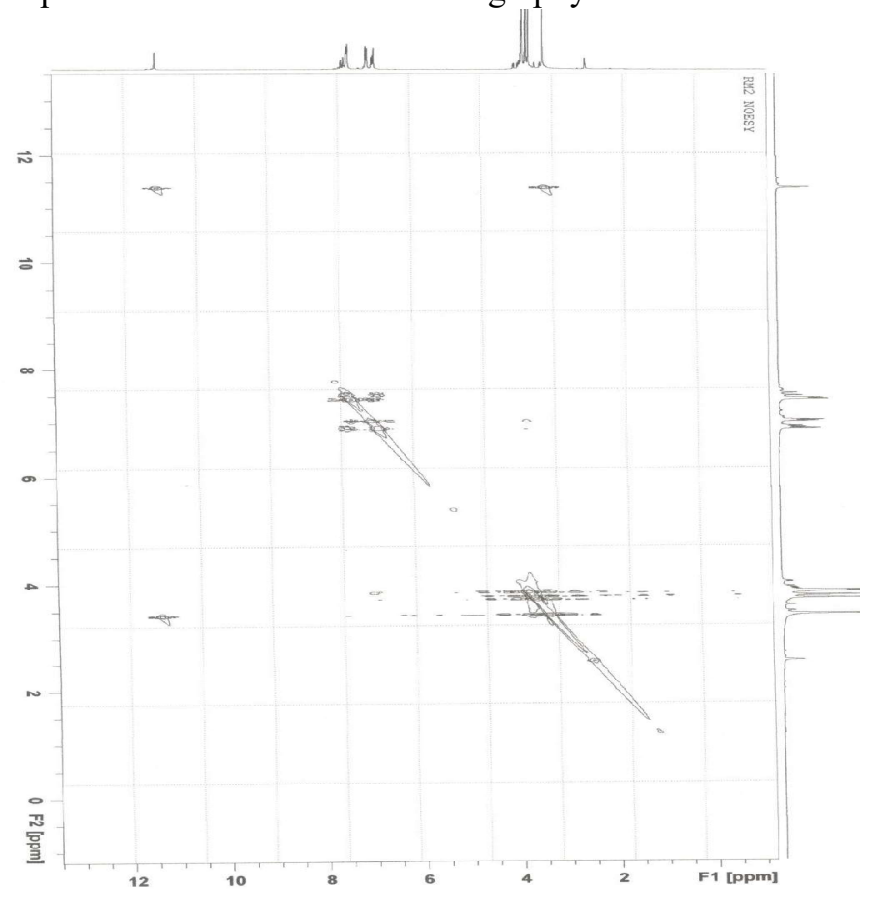

Figure 1. NOESY spectrum of compound 2k, DMSO-d 6 , $400 \mathrm{MHz}$ NMR.

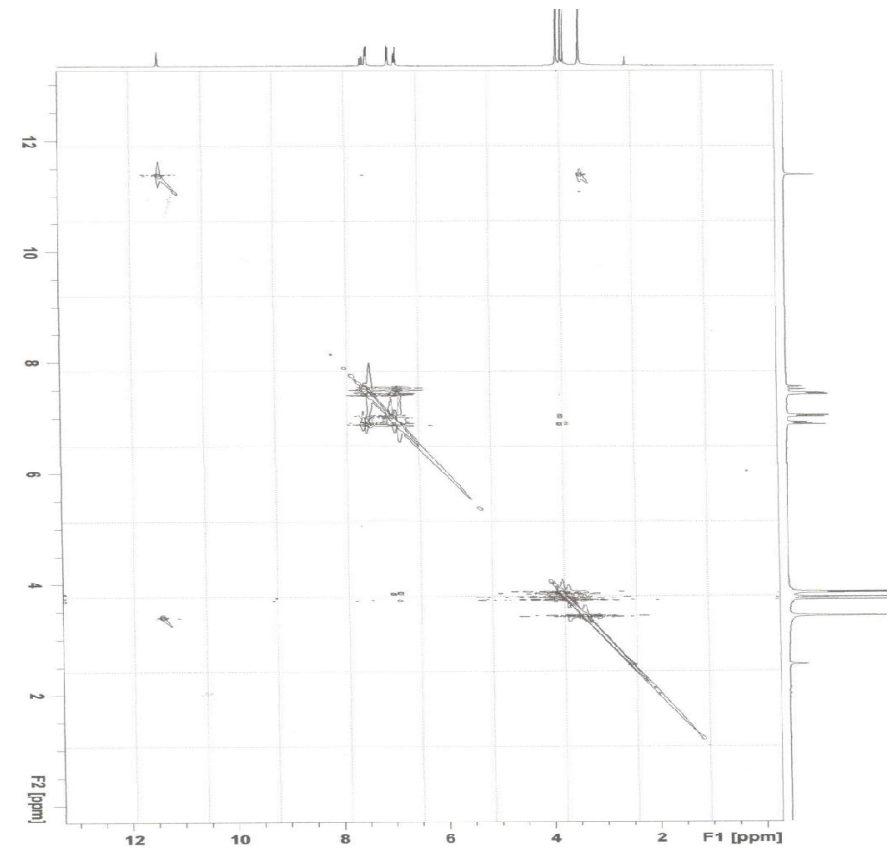

Figure 2. ROESY spectrum of compound $\mathbf{2 k}$, DMSO-d $\mathrm{d}_{6}, 400 \mathrm{MHz}$ NMR.

All the synthesized compounds (2a-2k) were characterized using spectroscopic techniques such as 1D-NMR: ${ }^{1} \mathrm{H},{ }^{13} \mathrm{C} / \mathrm{APT} / \mathrm{DEPT}$ and 2D-NMR: ${ }^{1} \mathrm{H}-{ }^{1} \mathrm{H}$ COSY, NOESY and ROESY, UV-Vis, FT-IR, LC-MS, elemental analyses and by the help of ACD NMR program. The mass spectra of chalcone oximes (2a-2k) showed molecular ion peaks at the appropriate $m / z$ values which are given in experimental part, respectively. The ${ }^{1} \mathrm{H}$ and ${ }^{13} \mathrm{C}$ NMR spectra of compounds $\mathbf{2 a - 2} \mathbf{k}$, in particular, $=\mathrm{NOH}$ showed peaks at within the range of $\delta_{\mathrm{H}} 11.02-11.48(1 \mathrm{H}, \mathrm{bs})$ and $\mathrm{C}_{1}$ at $\delta_{\mathrm{C}} 158.0 \mathrm{ppm}$, which are 
an indication of oxime group of chalcone. Also, the -OH moiety of products $\mathbf{2 a - 2} \mathbf{b}$ exhibited characteristic signals at $\delta_{\mathrm{H}} 9.7-9.8 \mathrm{ppm}$ in the ${ }^{1} \mathrm{H}$ NMR, respectively. The most noticeable feature for the structural characterization of chalcone oximes is the assignment of the proton resonances of the $\alpha, \beta$-unsaturated moiety within the range of $\delta 6.55-6.98 \mathrm{ppm}$ and $\delta$ 7.45-7.76 ppm with 15.6/16.0/16.4/16.8 Hz coupling constant, respectively which showed the stereochemistry of $\mathrm{CH}=\mathrm{CH}-$ in $E$ configuration. Structural elucidation for the geometry of chalcone oxime compounds was supported by a 2D NOESY (Figure 1) and ROESY (Figure 2) NMR spectra ${ }^{40}$ Here is the NOESY and ROESY spectra of compound $\mathbf{2} \mathbf{k}$ showed a spatial interaction between oxime $-\mathrm{OH}$ with $-\mathrm{OCH}_{3}$ at $\mathrm{C}-2$ position of $\mathrm{B}$ ring and $\beta-\mathrm{H}$ of the chalcone oxime compounds. Similar results were obtained for the other compounds. Therefore, geometry of compounds $\mathbf{2 a - 2} \mathbf{k}$ is assigned as $1 E, 2 E$-isomer in cisoid form.

In the literature, $1 Z, 2 E$ geometry of compounds $\mathbf{2} \mathbf{g}$ and $\mathbf{2} \mathbf{j}$ have mentioned. ${ }^{23}$ Moreover, their melting points were reported as $30{ }^{\circ} \mathrm{C}$ and $95{ }^{\circ} \mathrm{C}$, respectively. In our case, melting points of compounds $2 \mathrm{~g}$ and $\mathbf{2 \mathbf { j }}$ were found to be $113-114{ }^{\circ} \mathrm{C}(\mathbf{2 g})$ and $160-162{ }^{\circ} \mathrm{C}(\mathbf{2 j})$. These data clearly showed that compounds $\mathbf{2} \mathbf{g}$ and $\mathbf{2} \mathbf{j}$ were two different geometrical isomer $(1 Z, 2 E-/ 1 E, 2 E-)$ to each other. FT-IR spectrum of $\mathbf{2 a - 2 k}$ showed characteristic band of $=\mathrm{NOH}$ and $-\mathrm{OH}$ at $\sim 3165$ and $3285 \mathrm{~cm}^{-}$ ${ }^{1}$, and imine group $(-\mathrm{C}=\mathrm{N})$ at $1635-1658 \mathrm{~cm}^{-1}$, respectively.

\subsection{Theoretical Calculation of Compounds $\mathbf{2 a - 2 k}$}

The formation of the chalcone oxime can exist as geometric $E / Z$-isomers with four possible cisoid and transoid form (Figure 3).<smiles>[R]c1ccccc1OC</smiles>

$1 E, 2 E$ (cisoid form)<smiles>[R]c1ccccc1OC</smiles>

$1 Z, 2 E$ (cisoid form)<smiles>[R]c1cccc(OC)c1/C=C/C(=N/O)c1ccccc1</smiles>

$1 E, 2 E$ (transoid form)<smiles>[R]c1ccccc1/C=C/C(=N\O)c1ccccc1OC</smiles>

$1 Z, 2 E$ (transoid form)

Figure 3. The possible geometric isomer of the chalcone oxime compounds (2a-2k).

Table 1. Calculated energy for the geometrical isomers of compounds $\mathbf{2 a - 2 k}$ (PM3)

\begin{tabular}{ccccc}
\hline \multirow{2}{*}{ Compounds } & \multicolumn{2}{c}{$\begin{array}{c}\text { Conformation }(1 Z, 2 E) \\
(\mathrm{kcal} / \mathrm{mol})\end{array}$} & \multicolumn{2}{c}{$\begin{array}{c}\text { Conformation }(1 E, 2 E) \\
(\mathrm{kcal} / \mathrm{mol})\end{array}$} \\
& transoid & cisoid & transoid & cisoid \\
\hline $\mathbf{2 a}$ & 31.05 & 30.94 & 31.10 & 28.98 \\
$\mathbf{2 b}$ & 28.99 & 28.34 & 33.36 & 28.33 \\
$\mathbf{2 c}$ & 7.40 & 6.58 & 8.87 & 6.24 \\
$\mathbf{2 d}$ & -32.06 & -30.01 & -30.68 & -32.42 \\
$\mathbf{2} \mathbf{d}$ & -31.65 & -33.45 & -30.46 & -33.46 \\
$\mathbf{2 f}$ & -29.86 & -28.84 & -29.22 & -30.01 \\
$\mathbf{2 g}$ & -32.26 & -30.27 & -31.34 & -32.33 \\
$\mathbf{2 h}$ & -34.54 & -32.57 & -33.11 & -35.04 \\
$\mathbf{2 i}$ & -34.47 & -33.44 & -36.06 & -38.85 \\
$\mathbf{2 j}$ & -85.02 & -80.95 & -70.01 & -85.82 \\
$\mathbf{2} \mathbf{k}$ & -68.04 & -68.22 & -69.78 & -70.91 \\
\hline
\end{tabular}

Theoretical calculations were performed with HYPERCHEM 7.5 program on an HP PC intel core i5 computer. The molecular structures for the conformation of compounds $\mathbf{2 a - 2} \mathbf{k}$ were optimized with PM3 method. ${ }^{20-22}$. In the main skeletal structure of the molecules, conformational analyzes were performed over the six different torsional angel. An average of 120 conformation of each isomer was 
detected and the results are given in Table 1. The theoretical calculations of compounds $\mathbf{2 a - 2} \mathbf{k}$ showed that cisoid $1 E, 2 E$ is the most stable form of all for the compounds $\mathbf{2 a - 2 k}$. In the literature, the synthesis of different chalcone oxime compounds resulted $1 Z, 2 E$ and $1 E, 2 E$ geometrical isomers. ${ }^{23,34-}$ ${ }^{37,41}$ This could be due to the experimental condition which were used for the synthesis of chalcone oxime compounds.

\section{3. $\alpha$-Glucosidase Inhibitory Activity of Compounds $\mathbf{2 a - 2 k}$}

Synthesized chalcone oximes (2a-2k) were subjected to $\alpha$-glucosidase inhibition assay against acarbose. ${ }^{38-41}$ The $\mathrm{IC}_{50}$ values of substituted chalcone oximes (2a-2k) were listed in Table 2, and $\mathrm{IC}_{50}$ values of all these compounds were determined by interpolation of the dose-response curves and given as means of three experiments. No significant inhibitory effect was detected for compound $\mathbf{2} \mathbf{j}$ and $\mathrm{IC}_{50}$ values of compounds $\mathbf{2} \mathbf{i}-\mathbf{2} \mathbf{k}$ were not determined. Among the tested compounds, $\mathbf{2 a}$ and $\mathbf{2} \mathbf{b}$ showed the most significant $\alpha$-glucosidase inhibition activity by $51.52 \pm 1.09 \%$ and $47.24 \pm 6.08 \%$ at a concentration of $10 \mu \mathrm{M}$, respectively (Table 2). Acarbose, known $\alpha$-glucosidase inhibitor used as anti-diabetic drug, showed inhibitory effect by $43.27 \pm 1.91 \%$ at the same concentration. Compound $\mathbf{2 a}$ and $\mathbf{2 b} \mathrm{IC}_{50}$ values were calculated as $1.61 \pm 0.16 \mu \mathrm{M}$ and $3.36 \pm 0.58 \mu \mathrm{M}$, respectively.

Table 2. $\alpha$-Glucosidase inhibition of synthesized chalcone oximes $(\mathbf{2 a - 2 k})\left(\mathrm{IC}_{50}, \mu \mathrm{M}\right)$.

\begin{tabular}{crr}
\hline Compounds & Inhibition $\% \pm \mathrm{SD}(10 \mu \mathrm{M})$ & $\mathrm{IC}_{50}(\mu \mathrm{M}) \pm \mathrm{SD}$ \\
\hline $\mathbf{2 a}$ & $51.52 \pm 1.09$ & $1.61 \pm 0.16$ \\
$\mathbf{2 b}$ & $47.24 \pm 6.08$ & $3.36 \pm 0.58$ \\
$\mathbf{2 c}$ & $47.84 \pm 9.18$ & $11.26 \pm 2.04$ \\
$\mathbf{2 d}$ & $40.82 \pm 7.26$ & $9.20 \pm 2.90$ \\
$\mathbf{2 e}$ & $63.65 \pm 4.34$ & $9.39 \pm 0.94$ \\
$\mathbf{2 f}$ & $20.06 \pm 6.14$ & $12.58 \pm 0.98$ \\
$\mathbf{2 g}$ & $25.59 \pm 3.98$ & $14.00 \pm 2.00$ \\
$\mathbf{2 h}$ & $56.71 \pm 4.15$ & $25.55 \pm 0.38$ \\
$\mathbf{2 i}$ & $52.02 \pm 8.17$ & nd \\
$\mathbf{2 j}$ & $\mathrm{ni}$ & $\mathrm{nd}$ \\
$\mathbf{2} \mathbf{k}$ & $26.31 \pm 2.60$ & $\mathrm{nd}$ \\
Acarbose $^{\mathrm{a}}$ & $43.27 \pm 1.91$ & $13.34 \pm 1.26$ \\
\hline
\end{tabular}

ni: no inhibition, nd: Not determined, ${ }^{\mathrm{a}}$ Reference compound

Synthesized new chalcone oxime compounds $\mathbf{2 a}$ and $\mathbf{2 b}$ have substantial inhibition potential against the acarbose. When the methoxy is oriented at the ortho position of ring A of chalcone oximes, the number of methoxy substitution increased, $\alpha$-glucosidase inhibition activity of compounds $\mathbf{2 c - 2 e}$ increased with the $\mathrm{IC}_{50}$ values of 11.26 and $9.20 \mu \mathrm{M}$, respectively. Whereas, methoxy is substituted at the para positon of ring A of chalcone oximes, even increasing the number of methoxy substitution, $\alpha$ glucosidase inhibition activity of compounds $\mathbf{2 f - 2 l}$ decreased. Therefore, these result showed that the methoxy is at the ortho position of ring A is more effective than para position of chalcone oximes compounds for the $\alpha$-glucosidase inhibition activity. This result showed that the number of methoxy group might be an important effecting factor of inhibitory activities. When the methoxy group at the 3position of benzene ring (A) might be beneficial to improve this kind of compounds inhibitory activities that could be related to the lipophilicity. But, chalcone oxime (2a) had $-\mathrm{OH}$ at the meta positon of ring A gave the most $\mathrm{IC}_{50}$ value as $1.61 \pm 0.16 \mu \mathrm{M}$ (Table 2 and Figure 4). The $\alpha$-glucosidase inhibition activity indicated that chalcone oxime having hydroxyl group are more active than the chalcone oxime having methoxy group. Dose-dependent inhibitory effect of compounds $\mathbf{2 a}$ and $\mathbf{2 b}$ is shown in figure 4. 


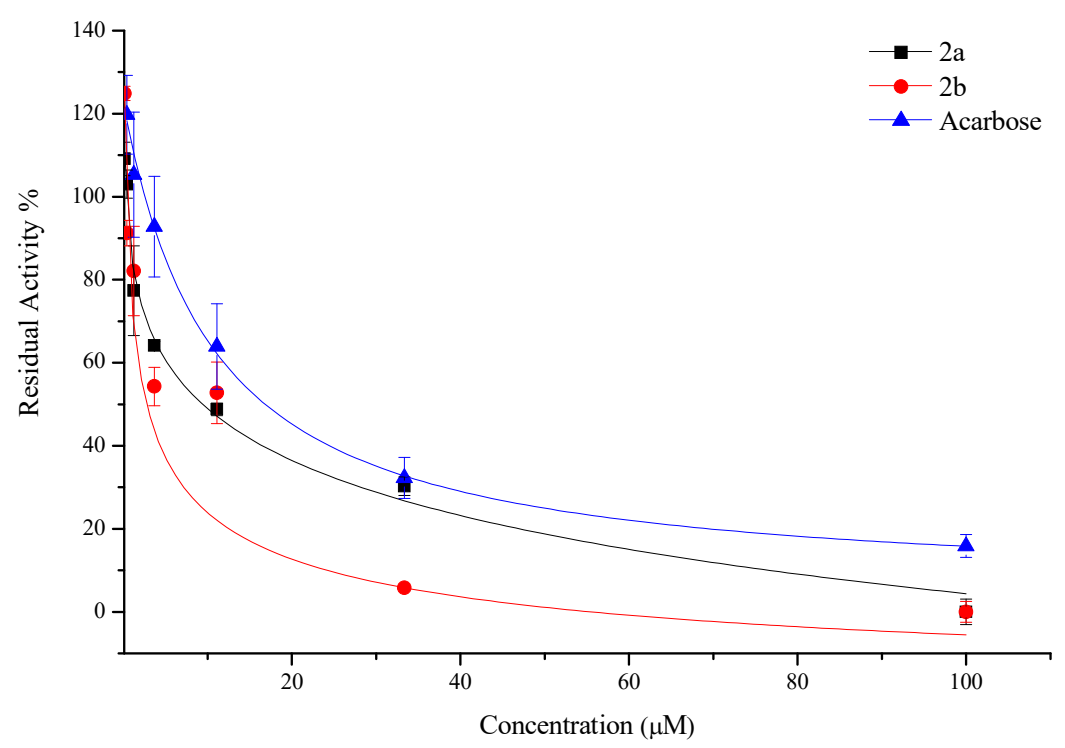

Figure 4. Dose-dependent inhibitory effect of compounds $\mathbf{2 a}$ and $\mathbf{2 b}$. Acarbose was used as standard inhibitor. Inhibitory effect of the tested compounds and Acarbose was measured at the range of $0.045-100 \mu \mathrm{M}$ concentrations. Residual activities of the compounds were expressed as the mean \pm S.D., measured in triplicate.

In the literature various substituted chalcone compounds such as; sulfonamide chalcone, ${ }^{42-43}$ 3 ',5'-digeranylated chalcone, ${ }^{44}$ aminochalcones, ${ }^{42}$ and the other substituted chalcone compounds ${ }^{42,45}$ were mentioned to be potent inhibitor against $\alpha$-glucosidase. In our case, screening of the hydroxyl and methoxy substituted chalcone oximes compounds (2a-2k) against $\alpha$-glucosidase revealed that hydroxyl substituted chalcone oximes $\mathbf{2 a}$ and $\mathbf{2 b}$ were found to be the best inhibitor against $\alpha$ glucosidase activity.

\section{Conclusion}

In summary, a two-step procedure for the synthesis of new hydroxyl and methoxy substituted chalcone oximes (2a-2k) from chalcone and hydroxylamine hydrochloride has been demonstrated. Synthetic chalcone oximes are very useful reactive intermediates for the efficient construction of various $N$-heterocycles and should be evaluated for their potential use of new bioactive chalcone oxime derivatives. Our results showed that most of compounds exhibited potent inhibition on $\alpha$ glucosidase inhibitory activity with $\mathrm{IC}_{50}$ values in the range of 1.61-25.55 $\mu \mathrm{M}$. Specifically, compounds bearing -OH (2a-2b) showed more potent inhibitory activities than the other compounds. In addition, compounds $\mathbf{2 a - 2 b}$ and $\mathbf{2} \mathbf{c}-\mathbf{2} \mathbf{g}$ demonstrated more potent inhibitory activity (1.61-12.58 $\mu \mathrm{M})$ than the reference standard inhibitor acarbose with $\mathrm{IC}_{50}$ value of $13.34 \mu \mathrm{M}$. Thus, most of the products exhibit good $\alpha$-glucosidase inhibition activity. Thus, compounds $2 \mathbf{2 a}, \mathbf{2 b}, \mathbf{2 e}, \mathbf{2 d}, \mathbf{2 c}, \mathbf{2 f}$ and $\mathbf{2 g}$ would be useful for the development of new drugs such as antidiabetics.

\section{Acknowledgements}

This study was supported by grants from Karadeniz Technical University Research Fund (KTUBAP 9699) of Turkey.

\section{Supporting Information}

Supporting information accompanies this paper on http://www.acgpbus.org/OC 


\section{ORCID}

$\begin{array}{ll}\text { Seda Fandaklı } & : \underline{0000-0002-8199-3336} \\ \text { İnci Selin Doğan } & : \underline{0000-0003-4949-1747} \\ \text { Hasan Erdinç Sellitepe } & : \frac{0000-0001-5339-6940}{: 0000-0002-5487-1536} \\ \text { Ahmet Yaşar } & : \underline{000-0003-4174-3014} \\ \text { Nurettin Yaylı } & \end{array}$

\section{References}

[1] Van Beers, E. H.; Büller, H. A.; Grand, R. J.; Einerhand, A. W. C.; Dekker, J. Intestinal brush border glycohydrolases: structure, function, and development. Crit. Rev. Biochem. Mol. Biol. 1995, 30, 197-262.

[2] Asano, N. Glycosidase inhibitors: update and perspectives on practical use. Glycobiology 2003, 13, 93R104R.

[3] Heightman, T. D.; Vasella, A. T. Recent Insights into Inhibition, Structure, and Mechanism of Configuration-Retaining Glycosidases. Angew. Chem. Int. Ed. 1999, 38, 750-770.

[4] Yee, H. S.; Fong, N. T. A review of the safety and efficacy of acarbose in diabetes mellitus. Pharmacotherapy 1996, 16, 792-805.

[5] Melo, E. B.; Gomes, A. S.; Carvalho, I. $\alpha$ - and $\beta$-Glucosidase inhibitors: chemical structure and biological activity. Tetrahedron 2006, 62, 10277-10302.

[6] Hwangseo, P.; Kyo Yeol, H.; Young Hoon, K.; Kyung Hwan, O.; Jae Yeon, L.; Keun K. Discovery and biological evaluation of novel $\alpha$-glucosidase inhibitors with in vivo antidiabetic effect. Bioorg. Med. Chem. Lett. 2008, 18, 3711-3715.

[7] Tadigoppula, N.; Gaurav, M.; Natasha, J.; Manali, A.; Chandan, K. M.; Neha, R.; Arvind, K. S.; Akhilesh, K. T. Synthesis of novel triterpene and $N$-allylated $/ N$-alkylated niacin hybrids as $\alpha$-glucosidase inhibitors. Eur. J. Med. Chem. 2013, 63, 162-169.

[8] Yuh-Meei, L.; Yasheen, Z.; Michael, T. F.; Li-Ming, Z.; Weiguo, N.; Fa-Ching, C. Chalcones and flavonoids as anti-Tuberculosis agents. Bioorg. Med. Chem. 2002, 10, 2795-2802.

[9] Sivakumar, P. M.; Prabu, Seenivasan, S.; Vanaja, K.; Mukesh, D. Synthesis, antimycobacterial activity evaluation, and QSAR studies of chalcone derivative. Bioorg. Med. Chem. Lett. 2007, 17, 1695-1700.

[10] Herencia, F.; Ferrandiz, M. L.; Ubeda, A.; Domínguez, J. N.; Charris, J. E.; Lobo, G. M.; Alcaraz, M. J. Synthesis and anti-inflammatory activity of chalcone derivatives. Bioorg. Med. Chem. Lett. 1998, 8, 1169-1174.

[11] Cheng, J. H.; Hung, C. F.; Yang, S. C.; Wang, J. P.; Won, S. J.; Lin, C. N. Synthesis and cytotoxic, antiinflammatory, and anti-oxidant activities of $2^{\prime}, 5^{\prime}$-dialkoxylchalcones as cancer chemopreventive agents. Bioorg. Med. Chem. 2008, 16, 7270-7276.

[12] Narender, T.; Khaliq, T.; Nishi, S.; Goyal, N.; Gupta S. Synthesis of chromenochalcones and evaluation of their in vitro antileishmanial activity. Bioorg. Med. Chem. 2005, 13, 6543-6550.

[13] Wu X.; Wilairat P.; Go M. L. Antimalarial activity of ferrocenyl chalcones. Bioorg. Med. Chem. Lett. 2002, 12, 2299-2302.

[14] Prasad, Y. R.; Rao, A. L.; Rambabu, R. Synthesis and Antimicrobial Activity of Some Chalcone Derivatives. E. J. Chem. 2008, 5, 461-466.

[15] López, S. N.; Castelli, M. V.; Zacchino, S. A.; Domínguez, J. N.; Lobo, G.; Charris-Charris, J.; Cortes, J. C. G.; Ribas, J. C.; Devia, C.; Rodríguez, A. M.; Enriz, R. D. In vitro antifungal evaluation and structureactivity relationships of a new series of chalcone derivatives and synthetic analogues, with inhibitory properties against polymers of the fungal cell wall. Bioorg. Med. Chem. 2001, 9, 1999-2013.

[16] Baviskar, B.; Patel, S.; Baviskar, B.; Khadabadi, S.S.; Shiradkar, M. Design and synthesis of some novel chalcones as potent antimicrobial agent. Asian J. Res. Chem. 2008, 1, 67-69.

[17] Nerya, O.; Musa, R.; Khatib, S.; Tamir, S.; Vaya, J. Chalcones as potent tyrosinase inhibitors: the effect of hydroxyl positions and numbers. Phytochemistry 2004, 65, 1389-1395.

[18] Liu, J.; Chen, C.; Wu, F.; Zhao, L. Microwave-assisted synthesis and tyrosinase inhibitory activity of chalcone derivatives. Chem. Biol. Drug. Des. 2013, 82, 39-47.

[19] Ya-Ting, L.; Tsorng-Harn, F.; Hui-Min, C.; Chao-Yuan, C.;Yun- Hsin, W.; Ching-Yuh, C.; Yau-Hung, C. Toxicity Assessments of Chalcone and Some Synthetic Chalcone Analogues in a Zebrafish Model. Molecules 2014, 19, 641-650. 
[20] Stewart J. J. P. Optimization of parameters for semiempirical methods I. Method., J. Comput. Chem., 1985, 101, 209-220.

[21] Stewart J. J. P. Semiempirical molecular orbital methods. In: rewiev in computational chemistry, Vol.1, Lipkowitz, K. B. and Boyd, D. B. (Eds.) VCH: New York; 45-81, 1990.

[22] Yaylı, N.; Yaşar, A.; Üçüncü, O.; Sivrikaya, S.Ö.; Güleç, C.; Küçük, M.; Abbasov, R., Synthesis of chalconoid-like compounds and their [2+2] photodimerizations in solution and theoretical calculations, $J$. Photochem. and Photobiol. A: Chem. 2005, 171, 291-298.

[23] Hailiang, Z.; Yanting, W.; Yajuan, Q. Chalcones oxime derivatives having inhibiting effect on cancer cell tubulin polymerization, and preparation method thereof, patent, CN103664689A, 20131104.

[24] Qiang, H.; Ji-Wei, W.; Hua-Jian, X. Biomimetic hydrogenation: a reusable NADH co-enzyme model for hydrogenation of $\alpha, \beta$-epoxy ketones and 1,2-diketones. Tetreh. Lett. 2013, 54, 3877-3881.

[25] Garg N.; Chandra T.; Archana, A. B.; Jain A.; Kumar A. Synthesis and evaluation of some new substituted benzothiazepine and benzoxazepine derivatives as anticonvulsant agents. Eur. J. Med. Chem. 2010, 45, 1529-1535.

[26] Ji W.C.; Bo K. J.; Nam-Chul C.; Jong-Hyun P.; Seul K. Y. ; Eun J. J.; Yong S. L.; Gyoonhee H.; Ae N. P.; Dong J. K.; Ki D. P. Synthesis of a series of unsaturated ketone derivatives as selective and reversible monoamine oxidase inhibitors. Bioorg. Med. Chem. 2015, 23, 6486-6496.

[27] Xiao-Guang, B.; Chang-Liang, X.; Shuang-Shuang, Z.; Hong-Wei, H.; Yu-Cheng, W.; Ju-Xian, W. Synthesis and cytotoxic evaluation of alkoxylated chalcones. Molecules 2014, 19, 17256-17278.

[28] Patil, P. S.; Bhumannavar, V. M.; Bannur, M. S.; Kulkarni, H. N.; Bhagavannarayana, G. F. Second harmonic generation in some donor-acceptor substituted chalcone derivatives. J. Cryst. Proc. Tech. 2013, 3, 108-117.

[29] Nunes, A. S.; Campos, V.P.; Mascarello, A.; Stumpf, T. R.; Chiaradia-Delatorre, L.D.; Teixeira Machado, A. R.; Santos Junior, H. M.; Yunes, R. A.; Nunes, R. J.; Oliveira, D. F. Activity of chalcones derived from 2,4,5-trimethoxybenzaldehyde against Meloidogyne exigua and in silico interaction of one chalcone with a putative caffeic acid 3-O-methyltransferase from Meloidogyne incognita. Exp. Parasitol. 2013, 135, 661-668.

[30] Wu, Jianzhang; Li, Jianling; Cai, Yuepiao; Pan, Yong; Ye, Faqing; Zhang, Yali; Zhao, Yunjie; Yang, Shulin; Li, Xiaokun; Liang, Guang. Evaluation and discovery of novel synthetic chalcone derivatives as anti-inflammatory agents. J. Med. Chem. 2011, 54, 8110-8123.

[31] Corrêa, M. J. C.; Nunes, F. M.; Bitencourt, H. R.; Borges, F. C.; Guilhon, G. M. S. P.; Arruda, M. S. P. ; Marinho, A. M. R.; Santo, A. S.; Alves, C. N; Brasil, D. S. B; Santos, L. S. Biotransformation of chalcones by the endophytic fungus Aspergillus flavus isolated from Paspalum maritimum Trin. J. Braz. Chem. Soc. 2011, 22, 1333-1338.

[32] Albay, C.; Kahriman, N.; Yılmaz, İskender, N.; Alpay, Karaoğlu, Ş.; Yaylı, N. Synthesis and antimicrobial activity of methoxy azachalcones and $\mathrm{N}$-alkyl substituted methoxy azachalconium bromides. Turk. J. Chem. 2011, 35, 441-454.

[33] Yaylı, N.; Üçüncü, O.; Yaşar, A.; Gök, Y.; Küçük, M.; Kolaylı S. Stereoselective Photochemistry Of Methoxy Chalcones In Solution And Their Radical Scavenging Activity. Turk. J. Chem. 2004, 28, 515521.

[34] Seo, W. D.; Kim, J. H.; Kang, J. E.; Ryu, H. W.; Curtis-Long, M. J.; Lee, H. S.; Yang, M. S.; Park, K. H. Sulfonamide chalcone as a new class of alpha-glucosidase inhibitors. Bioorg. Med. Chem. Let. 2005, 15, 5514-5516.

[35] Bharatham, K.; Bharatham, N.; Park, K. H.; Lee, K. W. Binding mode analyses and pharmacophore model development for sulfonamide chalcone derivatives, a new class of a-glucosidase inhibitors. J. Mol. Graph. and Model. 2008, 26, 1202-1212.

[36] Sun, H.; Li, Y.; Zhang, X.; Lei, Y.; Ding, W.; Zhao, X.; Wang, H.; Song, X.; Yao, Q.; Zhang, Y.; Ma, Y.; Wang, R.; Zhu T.; Yu P. Synthesis, $\alpha$-glucosidase inhibitory and molecular docking studies of prenylated and geranylated flavones, isoflavones and chalcones. Bioorg. Med. Chem. Let. 2015, 25, 4567-4571.

[37] Ansari, F. L.; Umbreen, S.; Hussain, L.; Makhmoor, T.; Nawaz, S. A.; Lodhi, M. A.; Khan, S. N.; Shaheen, F.; Choudhary M. I.; Rahman, A. Syntheses and biological activities of chalcone and 1,5benzothiazepine derivatives: promising new free-radical scavengers, and esterase, urease, and alphaglucosidase inhibitors. Chem. and Biodiv. 2005, 2, 487-496.

[38] Choudhary, M. I.; Adhikari, A.; Rasheed, S.; Bishnu, P. M.; Hussain, N.; Ahmad, K. W.; Rahman, A. Cyclopeptide alkaloids of Ziziphus oxyphylla Edgw as novel inhibitors of $\alpha$-glucosidase enzyme and protein glycation. Phytochem. Lett. 2011, 4, 404-406. 
[39] Seo, W. D.; Kim, J. H.; Kang, J. E.; Ryu, H. W.; Curtis-Long, M. J.; Lee, H. S.; Yang, M. S.; Park, K. H. Sulfonamide chalcone as a new class of alpha-glucosidase inhibitors. Bioorg. Med. Chem. Let. 2005, 15, 5514-5516.

[40] Bouhadir, K.; Atallah, H.; Mezher, R.; Fatfat, M.; Gali-Muhtasib, H.; Elaridi, J. Synthesis and biological assessment of novel acylhydrazone derivatives of 2-methyl-1,4-naphthoquinone. Org. Commun. 2017, 10, 259-272.

[41] Dangwal, K. L.; Semval, A. R. Microwave assisted synthesis and characterization of oxime derivatives of substitued chalcones. ICSR 2016, 5, 356-358.

[42] Liu, J.; Chen, C.; Wu, F.; Zhao, L. Microwave-assisted synthesis and tyrosinase inhibitory activity of chalcone derivatives. Chem. Biol. Drug Des. 2013, 82, 39-47.

[43] Wang, Y. T.; Qin, Y. J.; Zhang, Y. L; Li, Y. J.; Rao, B.; Zhang, Y. Q.; Yang, M. R.; Jiang, A. Q.; Qi, J. L.; Zhu, H. L. Synthesis, biological evaluation, and molecular docking studies of novel chalcone oxime derivatives as potential tubulin polymerization inhibitors. $R S C A d v$. 2014, 4, 32263-32275.

[44] Desai, V. G.; Naik, S. R.; Dhumaskar , K. L. o-Iodoxy benzoic acid-mediated synthesis of 3,5diarylisoxazoles and isoxazole-3-carboxylic acids. Synth. Commun. 2014, 44, 1453-1460.

[45] Luo, Y.; Song, R.; Li, Y.; Zhang, S.; Liu, Z. J.; Fu, J.; Zhu, H. L. Design, synthesis, and biological evaluation of chalcone oxime derivatives as potential immunosuppresive agents. Bioorg. Med. Chem. Lett. 2012, 22, 3039-3043.

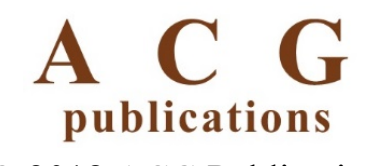

(C) 2018 ACG Publications 